\title{
Intestinal microbiota in digestive diseases
}

\author{
Maria do Carmo Friche PASSOS' ${ }^{1}$ and Joaquim Prado MORAES-FILHO²
}

Received 23/2/2017 Accepted 14/3/2017

\begin{abstract}
Background - In recent years, especially after the development of sophisticated metagenomic studies, research on the intestinal microbiota has increased, radically transforming our knowledge about the microbiome and its association with health maintenance and disease development in humans. Increasing evidence has shown that a permanent alteration in microbiota composition or function (dysbiosis) can alter immune responses, metabolism, intestinal permeability, and digestive motility, thereby promoting a proinflammatory state. Such alterations can mainly impair the host's immune and metabolic functions, thus favoring the onset of diseases such as diabetes, obesity, digestive, neurological, autoimmune, and neoplastic diseases. This comprehensive review is a compilation of the available literature on the formation of the complex intestinal ecosystem and its impact on the incidence of diseases such as obesity, non-alcoholic steatohepatitis, irritable bowel syndrome, inflammatory bowel disease, celiac disease, and digestive neoplasms. Conclusion - Alterations in the composition and function of the gastrointestinal microbiota (dysbiosis) have a direct impact on human health and seem to have an important role in the pathogenesis of several gastrointestinal diseases, whether inflammatory, metabolic, or neoplastic ones.

HEADINGS - Microbiota. Gastrointestinal microbiome. Dysbiosis. Gastrointestinal diseases.
\end{abstract}

\section{INTRODUCTION}

The human gastrointestinal microbiota ("microflora") consists in a group of microorganisms that live in the digestive tract. They comprise a metabolically active and complex ecosystem, consisting of hundreds of thousands of microorganisms (bacteria, viruses, and some eukaryotes) that colonize the digestive tract soon after birth $^{(11,20)}$. The microbiota has established a dynamic association of mutual benefits (symbiosis) with the human organism, which results in the maintenance of normal immunological, metabolic, and motor functions, as well as correct nutrient digestion and absorption ${ }^{(11,20,30,53)}$.

The microbiota acts as a true barrier to aggressive agents, as more than $10^{14}$ microorganisms cover the entire surface of the digestive tract, primarily in the intestine, competing with pathogens for nutrients and binding sites, producing inhibitory substances and preventing their penetration into the intestinal mucosa ${ }^{(30,42,59)}$. This population encodes 3 to 4 million genes, or approximately 150 times more than the human genome ${ }^{(20,42)}$. As it will be discussed below, the microbial genome allows the microbiota agents to perform several metabolic activities that are not encoded by the human genome and, therefore, can be beneficial to the host.

Humans have their own individual pattern of microbiota distribution and composition, which is in part determined by the host genotype and by the initial colonization that occurs immediately after birth ${ }^{(59)}$. Different factors such as the type of delivery and breastfeeding, lifestyle, diet, hygienic and environmental conditions, antibiotic use, and vaccination can determine definitive changes in the microbiota pattern ${ }^{(9,46,59)}$.

The intestinal microbiota consists of more than one thousand and five hundred species, distributed in more than 50 different phyla ${ }^{(20,53)}$, although most are represented by only two phyla: Firmicutes (more abundant) and Bacteroidetes. Other phyla also found in minor proportions are Proteobacteria, Actinobacteria, Fusobacteria and Verrucomicrobia ${ }^{(53)}$.

An important European study (the "MetaHit project") analyzed the intestinal microbiota of 700 healthy volunteers, showing that the participants' microbiota composition, regardless of age, gender and body mass index, belonged to one of the three major groups or types, called enterotypes ${ }^{(33)}$. Each enterotype was identified based on the abundance of bacteria in relation to three main genera: Bacteroides (enterotype 1), Prevotella (enterotype 2) and Ruminococcus (enterotype 3$)^{(5,33)}$. However, it is not known what factors promote the aggregation of bacterial communities into enterotypes and it is believed that there may be a constant variation of these species in the digestive $\operatorname{tract}^{(5,42)}$.

The distribution of the intestinal microbiota varies according to its location in the digestive tube ${ }^{(20,53)}$. In the stomach and duodenum, due to the presence of acidic gastric juice and pancreatic enzymes, the bacterial density is quite low. It gradually increases in the distal small intestine, reaching its highest concentration $\left(10^{11}-10^{13} \mathrm{bacteria} / \mathrm{g}\right)$ in the colon, with the absolute predominance of anaerobes ${ }^{(11,20)}$.

In recent years, especially after the development of complex metagenomic studies, research in this area has increased, radically transforming our knowledge on the intestinal microbiota and its association with human health maintenance.

Increasing evidence has shown that a permanent alteration in the microbiota composition or function (dysbiosis) can alter visceral sensitivity, intestinal motility, and permeability, as well as alter the immune response, thus promoting a proinflammatory state ${ }^{(4,53)}$. Such alterations, especially in the host's immune and metabolic

Declared conflict of interest of all authors: non

Disclosure of funding: no funding received

'Faculdade de Medicina da Universidade Federal de Minas Gerais; Instituto Alfa de Gastroenterologia, Belo Horizonte, MG, Brasil; ${ }^{2}$ Faculdade de Medicina da Universidade de São Paulo, SP, Brasil.

Correspondence: Maria do Carmo Friche Passos. Rua Piauí, 69/804, Santa Efigênia - CEP: 30150-320 - Belo Horizonte, MG, Brasil. E-mail: mcpassos.ufmg@gmail.com 
functions, can originate or favor the onset of several diseases such as diabetes, obesity, as well as neurological and autoimmune diseases $^{(42,54)}$. Recent studies have also demonstrated the participation of the microbiota in the etiopathogenesis of many gastroenterological diseases, such as irritable bowel syndrome, inflammatory bowel disease, celiac disease, non-alcoholic steatohepatitis and digestive neoplasms ${ }^{(22,34)}$.

\section{Microbiota and Immunity}

The intestinal microbiota exerts an important effect on the immune response of humans, being crucial for the development and expansion of lymphoid tissues and for the maintenance and regulation of intestinal immunity ${ }^{(11,20,30,53)}$.

Experimental studies on germ-free mice have shown that bacterial colonization is a fundamental condition for the development of gut-associated lymphoid tissue (GALT), as well as for the diversified formation of B-cell $\operatorname{IgM}^{+4}$ antibodies ${ }^{(46,59)}$. It was observed that the intestinal mucosa of these non-colonized animals had a lower number of $\mathrm{B}, \mathrm{T}$ and dendritic cells, as well as immature mesenteric lymph nodes and Peyer's patches ${ }^{(59)}$. Additionally, their villi are narrower and longer and the crypts are deeper. It has been The authors emphasized that after being colonized by commensal microorganisms, the immune system of these mice develops and normalizes (Figure 1).

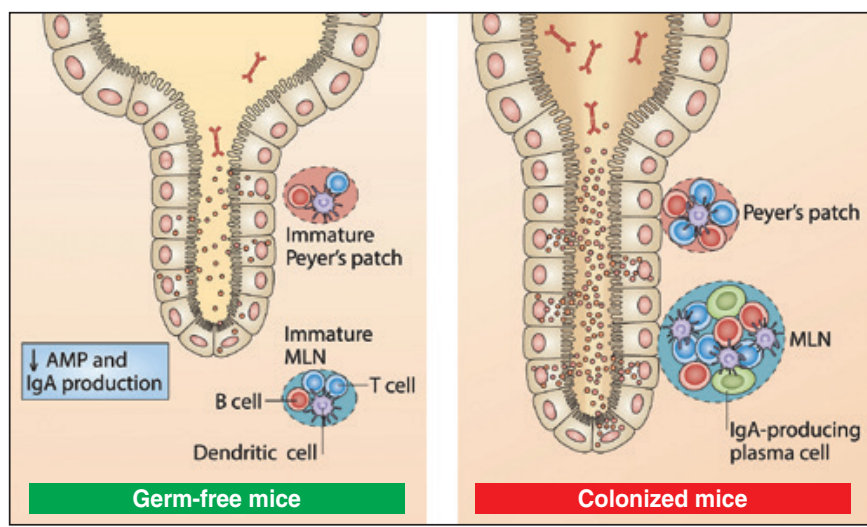

FIGURE 1. Immune system development after bacterial colonization in germ-free experimentation animals. Adapted from Sommer and Bäckhed, $2013^{(59)}$.

The induction of the immunological tolerance in the intestine is essential and as such no undesirable inflammatory responses occur against food proteins or even against the intestinal microbiota ${ }^{(20,53)}$. $\mathrm{T}$ cells can generate subpopulations of which immune responses may be anti or proinflammatory ${ }^{(59)}$.

Bacteroides fragilis is an example of bacteria present in the intestine, capable of inducing the differentiation of CD4+ T cells into regulatory $\mathrm{T}$ cells, which favors the production of anti-inflammatory cells and the transforming growth factor beta (TGF- $\beta$ ), neutralizing the pro-inflammatory response of Th17 (T helper) ${ }^{(62)}$. The capsule of this bacterium consists of polysaccharide A.

On the other hand, the group of filamentous bacteria, after contacting the antigen-regulating cells, seems capable of inducing pro-inflammatory cells such as Th1 $7^{(44)}$. Obviously, inflammation induction, when well controlled, may even be beneficial and even necessary. Some authors suggest that the immunological responses triggered by the intestinal microbiota can strengthen the intestinal barrier $^{(11,20,44)}$. In fact, mice that do not have filamentous bacteria have a more fragile immune response and, consequently, become more susceptible to infections ${ }^{(4,54,62)}$.

All these findings from recent studies suggest that the intestinal microbiota and the immune system establish a constant interaction of mutualism with the host, in which both are benefited ${ }^{(11)}$. This interrelationship results in several immunological responses, such as immunoglobulin A secretion and the release of antimicrobial peptides, which allow the maintenance of a dynamic equilibrium with the commensal microorganisms.

Recent studies have confirmed the initial hypotheses that the decrease in the microbial population caused by the improvement of hygienic conditions may have contributed to the increase in the prevalence of autoimmune diseases in developed countries ${ }^{(61)}$. In fact, dietary and environmental alterations can lead to qualitative and quantitative changes in the intestinal microbiota and immune response and, therefore, can eventually contribute to the increased incidence of diseases with a significant inflammatory component such as asthma, obesity and diabetes ${ }^{(61,67)}$. It is concluded that a healthy diet contributes to the growth of bacteria that are beneficial to the host. An important characteristic in this case is the presence in the diet of substances that can be employed by bacteria, such as resistant starch fibers, found in fruits, vegetables, and garlic. These foods are more resistant to the high digestive enzymes found in the digestive $\operatorname{tract}^{(10)}$.

\section{Microbiota and metabolism}

The intestinal microbiota contributes directly to the metabolization of nutrients and vitamins essential for host viability, collaborating to obtain energy from food ${ }^{(11,20,53)}$. This energy is acquired especially through the fermentation of non-absorbable carbohydrates, in a reaction that induces the production of shortchain fatty acids (SCFAs), hydrogen and carbon dioxide ${ }^{(44,62)}$.

Current research demonstrates that SCFAs have much more refined functions than merely nourishing microorganisms and enterocytes, and are now considered important regulators of immunity, energy metabolism and adipose tissue expansion. Fatty acids can activate the G-protein coupled receptors - GPR41 and GPR43 - expressed in several cells (immune, endocrine, and adipose cells) ${ }^{(21)}$. This process is important for the YY peptide expression and the glucagon-like peptide 1 (GLP-1) production, directly contributing to lipolysis inhibition and adipocyte differentiation, with consequent increase in adipose tissue in animals. The SCFAs also allow the acidification of the colonic lumen, preventing the growth of bacterial pathogens ${ }^{(21,35)}$.

It is important to emphasize that the intestinal microbiota has a direct participation in the metabolism of bile acids from dietary cholesterol. In the gut, primary bile acids bind to cell receptors, promote the absorption of fat and fat-soluble vitamins, and bind to cell receptors, such as TGR-5, which, when activated, trigger several protective metabolic effects such as resistance to weight gain and the development of hepatic steatosis ${ }^{(27)}$.

The anaerobic metabolism of bacteria also includes proteolytic fermentation in the distal colon, originating nitrogenous derivatives such as amines and ammonia, some of which have carcinogenic effects $^{(43)}$.

\section{Microbiota, obesity, and non-alcoholic steatohepatitis}

Obesity arises mainly due to the consumption of high-calorie foods, carbohydrates, and saturated fats, although the simple 
increase in caloric intake is not enough to explain the current obesity epidemic. Studies on germ-free mice showed that they did not increase their weight when exposed to high-calorie diets, from which one infers that diet alone is not enough to induce obesity ${ }^{(35)}$. On the other hand, obese mice have more genes encoding enzymes that break down nondigestible polysaccharides from the diet, in addition to having more fermentation products (SCFAs) and fewer calories in their feces, suggesting that in these animals the microbiota seems to help by extracting additional calories from the diet ${ }^{(65)}$. Other studies also found that germ-free mice, after being colonized, exhibited not only increased total body fat (despite a lower caloric intake), but many of them became insulin-resistant ${ }^{(14,65)}$.

The intestinal microbiota also participates in the digestion of polysaccharides, increasing the amount of glucose in the liver and, therefore, increasing lipogenesis ${ }^{(14)}$.

An "obese type" human microbiota associated with metabolic syndrome and overweight has been described, which shows an increase in the Firmicutes/Bacteroidetes ratio ${ }^{(29,42,65)}$. It has been shown that genetically obese mice have $50 \%$ fewer Bacteroidetes and more Firmicutes than lean animals ${ }^{(13,65)}$. It has been proven by several researchers that the microbiota of obese animals releases more calories during digestion than that of lean animals. It is also noteworthy that the administration of a typical high-calorie western diet in normal weight animals determines a marked reduction of Bacteroidetes and an increase of Firmicutes ${ }^{(22,30,42)}$.

Recent studies suggest that Bifidobacteria and Bacteroides ssp seem to be able to protect against weight gain, giving rise to the "microbial hypothesis" for obesity, which may determine important therapeutic implications in the future ${ }^{(13,14,32)}$.

In summary, the multiple metabolic mechanisms that associate

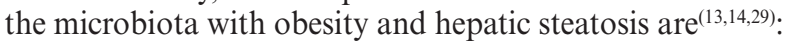

1) Bacterial fermentation of polysaccharides that are not digested by man, which leads to the production of monosaccharides and short-chain fatty acids (SCFAs). These are substrates of colonocytes and precursors of cholesterol and fatty acids, which also participate in hepatic gluconeogenesis;

2) SCFAs bind to specific receptors in intestinal endocrine cells, increasing the YY peptide, capable of reducing appetite by slowing intestinal transit and increasing nutrient absorption and leptin levels;

3) Microbial regulation of some host genes that promote lipid deposition in adipocytes;

4) Reduction of the intestinal expression of the adipose factor induced by fasting, thus favoring the accumulation of fatty acids and adipose tissue expansion;

5) Increased hepatic capture of monosaccharides from the portal circulation with activation of substances involved in lipogenesis regulation;

6) Vascularization induced by inflammation and mucosal blood flow (stimulated by the intestinal microbiota), increasing nutrient absorption;

7) Promotion of a low-grade pro-inflammatory state, as well as increased insulin resistance and cardiovascular risk, through mechanisms involving exposure to bacterial products, especially lipopolysaccharides produced by gram-negative bacteria. This process is called "metabolic endotoxemia"(24).

In conclusion, the microbiota is potentially capable of affecting both sides of the energy balance, influencing energy acquisition from diet components and altering some host genes, which regulate energy consumption and storage.

\section{Microbiota and Digestive Diseases Irritable Bowel Syndrome}

Although the etiopathogenesis of Irritable Bowel Syndrome (IBS) is not fully understood, several pathophysiological changes have been described (altered intestinal motility, visceral hypersensitivity, immune activation, dysregulation of the brain-bowel axis), thus constituting a multifactorial syndrome ${ }^{(39)}$. In recent years, important etiopathogenic contributions have been described, with the demonstration that some patients with IBS also have an alteration in the microbiota and changes in the intestinal mucosa (dysbiosis) ${ }^{(39,51,58)}$.

The most relevant evidence supporting a possible participation of the microbiota in the pathophysiology of IBS are ${ }^{(8,40)}$ :

a) Frequently observed qualitative and quantitative alterations in the intestinal microbiota;

b) Subgroup of patients has a history of acute gastroenteritis preceding the chronic symptom onset, being considered in this case as having post-infection IBS;

c) Higher prevalence of intestinal bacterial overgrowth when compared to healthy controls;

d) Dietary restriction of fermentable oligosaccharides, disaccharides, monosaccharides, and polyols (FODMAPs) often attenuates intestinal;

e) Possible modulation of the intestinal microbiota with antibiotics, probiotics and prebiotics and clinical improvement.

\section{a) Qualitative and quantitative changes in intestinal microbiota in IBS}

Initial studies of the intestinal microbiota in patients with IBS demonstrated a decrease in the proportion of Bifidobacterium and Lactobacillus and an increase in Enterobacter ${ }^{(67)}$. A greater reduction of Lactobacillus was detected in patients with IBS and diarrhea (IBS-D) than in those with IBS and constipation (IBS-C) ${ }^{(51)}$. It was also observed that a lower amount of Bifidobacteria is found in SII-D and a greater amount of Veillonella is found in IBS-C than in the control groups ${ }^{(57)}$. On the other hand, there is evidence of higher levels of Enterobacteriaceae and lower of Faecalibacterium prausnitzii in patients with IBS-D than in healthy controls ${ }^{(16)}$.

Using DNA sequencing techniques, 24 fecal samples from patients with IBS and 23 controls were analyzed, confirming a decrease in the proportion of Lactobacillus and Bifidobacteria ${ }^{(36)}$. Another study observed a two-fold increase in the Firmicutes/ Bacteroidetes ratio in patients with IBS $(P<0.0002)$, in addition to an association between abdominal pain and low amounts of Bifidobacteria also being reported during a 7-week follow-up ${ }^{(52)}$.

Research on experimental animals have demonstrated that behavioral changes, such as stress, can change the composition of the intestinal microbiota, making it more vulnerable to the inflammatory and immunological stimuli of the gastrointestinal tract ${ }^{(39,51)}$. It is worth mentioning that the great heterogeneity of the results is justified, at least in part, by the multiple methods used to determine the microbiota and the different inclusion criteria used for patients with IBS (Roma II/III).

\section{b) Post-infection IBS and bacterial overgrowth of the small intestine}

A strong indication of the importance of microbiota and lowgrade inflammation in the etiology of IBS is the onset of chronic IBS-compatible symptoms after acute gastroenteritis ${ }^{(39)}$. Postinfection IBS has been associated with several microorganisms, including Campylobacter jejuni, Yersinia, Salmonella and Shigella ${ }^{(60)}$. 
More recent evidence indicates that, in addition to bacteria, viral infections (rotavirus, adenovirus, calicivirus) and parasitic infections (Giardia lamblia, Blastocystis hominis) may also be involved in the onset of this syndrome ${ }^{(58)}$.

Epidemiological data published in recent years suggest that up to $20 \%$ of individuals with gastroenteritis will develop post-infection IBS and that there are factors considered to be of risk for its onset, such as: female gender, younger age, microorganism toxicity, prolonged diarrhea, and presence of stressful psychological events during the infection (e.g., anxiety and depression $)^{(60)}$. The severity of the initial condition (based on the need to seek emergency care and hospitalization) is the main predictive factor for the subsequent development of a chronic IBS-compatible condition ${ }^{(40)}$.

A meta-analysis showed the presence of IBS after acute gastroenteritis in $9.8 \%$ of the patients $(4.0-13.3)$ when compared to $1.2 \%$ in the control groups $(0.4-1,8 ; P=0.01)$, showing that the relative risk of developing IBS was 7.3-fold higher after an episode of acute intestinal infection ${ }^{(31)}$. Another meta-analysis observed a nearly six-fold increase in the estimated risk of developing IBS after gastroenteritis and that this risk remains elevated up to 3 years after the acute infection ${ }^{(63)}$.

In a well-conducted systematic review and meta-analysis including six studies, the presence of IBS after traveler's diarrhea was demonstrated in $5.4 \%$ of patients $(2.35-6.49)$ compared to $1.4 \%$ in the control groups $(P<0.00001)$. It was concluded that the relative risk of developing IBS after an episode of traveler's diarrhea (which can be caused by bacteria, mainly Escherichia coli, viruses, and parasites) is on average three to four-fold higher ${ }^{(55)}$.

There is important evidence that the intestinal microbiota is altered in this subgroup of patients and that there is mucosal microinflammation characterized by cell infiltration, alteration in the number of mast cells and T lymphocytes, mRNA abnormalities of interleukin-1 (IL-1), reduction in the IL-10/IL-12 ratio, increased circulation of IL-6, IL-8 and tumor necrosis factor-alpha ${ }^{(8,52)}$.

Considering the abovementioned facts, researchers believe that post-infection IBS is often accompanied by bacterial overgrowth in the small intestine (BOSI) ${ }^{(60)}$. There is, in fact, a great overlap of symptoms in the two syndromes - diarrhea, constipation, abdominal pain, flatulence, and abdominal distension - and therefore, the possibility that IBS patients have underlying BOSI has been questioned.

A relevant study carried out by researchers from Los Angeles, USA, found a clear association between the presence of bacterial overgrowth and reduction of the interstitial cells of Cajal, which could explain the development of post-infectious IBS ${ }^{(48)}$. Additionally, it has been confirmed in different studies that the prevalence of overgrowth is significantly higher in patients with IBS than in healthy controls ${ }^{(47)}$.

\section{c) Efficacy of a diet poor in FODMAPs (fermentable oligosaccharides, disaccharides, monosaccharides, and polyols) and intestinal microbiota modulatory therapies in relieving IBS symptoms}

It is believed that the products of bacterial fermentation may be implicated in the etiopathogenesis of $\operatorname{IBS}^{(39,58)}$. Increased fermentation can cause an increase in gas production, triggering the onset of typical symptoms of the syndrome, such as flatulence, abdominal pain, and distension. It has been reported that SCFA concentrations are increased in IBS-D and that they can stimulate serotonin release from the intestinal mucosa, with consequent increase in intestinal motility and transit ${ }^{(28)}$.
FODMAPs are short-chained carbohydrates that are poorly absorbed in the small intestine and are used in the colon as a substrate for bacterial fermentation. These carbohydrates also have an osmotic effect, because they cause a displacement of water to the intestinal lumen. The effect of the FODMAP-rich diet on the onset of IBS symptoms seems to be directly associated with the intestinal microbiota composition, suggesting that its manipulation may constitute an effective therapeutic pathway in the treatment of IBS. In fact, several recent studies have shown that a diet poor in FODMAPs is effective in relieving IBS symptoms, especially flatulence and diarrhea, constituting an effective approach for patients with functional intestinal symptoms ${ }^{(28,39)}$. (Figure 2).

\begin{tabular}{|c|c|}
\hline Category & High FODMAP content \\
\hline Vegetables & $\begin{array}{c}\text { Asparagus, artichoke, onion, } \\
\text { garlic, legumes, peas, beets, corn, } \\
\text { beans }\end{array}$ \\
\hline Fruits & $\begin{array}{c}\text { Apple, pear, mango, watermelon, } \\
\text { plum }\end{array}$ \\
\hline Milk/ Dairy products & Milk, yogurt, white cheese \\
\hline Bread / Cereals & $\begin{array}{c}\text { Made with wheat, rye; pasta, } \\
\text { pizza }\end{array}$ \\
\hline Cookies/Biscuits & Made with wheat, rye, barley \\
\hline Seeds / Nuts & Pistachios, cashew nuts, walnuts \\
\hline
\end{tabular}

FIGURE 2. Examples of foods with high FODMAP content.

Evidence has suggested that therapies capable of modulating the intestinal microbiota (antibiotics, probiotics and prebiotics) may improve symptoms by reinforcing the role of dysbiosis in the syndrome pathogenesis ${ }^{(28,38,39)}$.

Several clinical trials have shown that a significant portion of patients with IBS who have bacterial overgrowth improve with antibiotic therapy. A well-conducted double-blind, randomized study compared rifaximin and placebo in patients with IBS, showing an overall symptom improvement, especially flatulence in the group receiving the active drug ${ }^{(48)}$. Another study showed that a single treatment with rifaximin for 7 days may improve IBS symptoms in $46 \%-90 \%$ of patients and normalize the respiratory test in 20 $75 \%$ of cases $^{(49)}$.

Rifaximin is not yet available in Brazil and the most commonly used antibiotics in our country for these cases are quinolones, metronidazole, amoxicillin-clavulanic acid, and tetracycline.

Significant improvement in abdominal distension and pain has been reported with the use of probiotics containing Bifidobacterium infantis $35624^{(38)}$. It has also been shown that Lactobacillus supplementation is associated with decreased gas-production related symptoms in patients with IBS ${ }^{(24)}$.

\section{d) Probiotics in IBS}

Probiotics are living microorganisms that, when consumed in adequate amounts, can contribute to good health ${ }^{(45)}$. However, many questions regarding its use of probiotics in gastrointestinal disorders remain to be answered, such as most optimal doses, duration of treatment, physiological and immunological effects and safety in debilitated patients. Even so it is interesting to consider the crescent number of studies on the role of probiotics in different gastrointestinal disorders and the trend to consider them as an important adjuvant therapy. 
The use of probiotics in the IBS is an example of this approach: a systematic review including 19 randomized controlled trials concluded that probiotics are superior to placebo in relieving the IBS symptoms ${ }^{(24)}$.

The mechanism of action of probiotics seems to be broader than simply modulating the intestinal microbiota. It is possible that they also act to inhibit the colonization and adherence of pathogenic bacteria to the enterocytes, increasing the secretion of defensins and decreasing the synthesis of pro-inflammatory cytokines, such as IL-10 and IL-12 $2^{(47)}$. The figure below is only demonstrative and may not include all the species/strains currently available on the market. (Figure 3).

\begin{tabular}{|c|}
\hline Lactobacillus acidophilus NCFM \\
\hline Lactobacillus acidophilus $\mathrm{LA5}$ \\
\hline Lactobacillus rhamnosus $\mathrm{R} 0011$ \\
\hline Lactobacillus reuteri $\mathrm{RC}-14$ \\
\hline Lactobacillus casei shirota \\
\hline Saccharomyces cerevisae boulardi \\
\hline Bacillus coagulans BC3 \\
\hline Bifidobacterium animalis lactis Bi-07 \\
\hline Bifidobacterium lactis Bb-12 \\
\hline Bifidobacterium bifidum CUL 20 \\
\hline
\end{tabular}

FIGURE 3. Examples of probiotics.

\section{Inflammatory Bowel Diseases (IBD)}

Environmental factors, genetic factors and immune responses have been considered as the major etiology of IBD (Crohn's Disease and Ulcerative Colitis) which have a diversified pathogenesis. However, in recent years researchers have been very interested in evaluating the possible involvement of the intestinal microbiota in the complex etiopathogenesis of IBD ${ }^{(10,30,42}$. The main evidence that supports a possible participation of the microbiota in the pathophysiology of IBD are shown in Figure $4^{(7,15)}$.

Qualitative and quantitative alterations in intestinal microbiota

Occurrence of lesions in intestinal segments with higher concentrations of bacteria

Polymorphisms of genes encoding bacterial receptors

Experimental "germ free" animals do not develop ulcerative colitis

Increased intestinal permeability in Crohn's disease

FIGURE 4. Participation of the microbiota in the pathophysiology of IBD - main evidence.

Initial studies, based on stool cultures, observed a significant decrease in the intestinal microbiota biodiversity in patients with $\mathrm{IBD}^{(7)}$. Obviously, only alterations in diversity would not be the cause of the inflammatory disease, with a susceptible genotype being indispensable, which occurs in the presence of specific mutations ${ }^{(15,42)}$. A reduction in the Bacteroidetes and Firmicutes phyla and the concomitant increase in Proteobacteria and Actinobacteria have been described ${ }^{(7)}$. There is also a tendency for the excess of pro-inflammatory organisms with concomitant depletion of organisms with anti-inflammatory properties, such as Faecalibacterium prausnitzii $3(30,42,53)$ - (Figure 4$)$.

Studies have shown an increase in Enterobacteriaceae ( $E s$ cherichia coli and Shigella) in IBD ${ }^{(18,19)}$. Colonization by E. coli with adherent and invasive properties is more frequent in the ileal mucosa of patients with Crohn's disease than in healthy controls ${ }^{(19)}$. This group of bacteria has been identified more frequently in the stool of patients with active $\operatorname{IBD}^{(7,18,19)}$.

Studies have shown that there is a higher concentration of bacteria of the genus Fusobacterium in the colonic mucosa of patients with ulcerative colitis ${ }^{(7,42)}$. Additionally, it has been emphasized in experimental studies that the Fusobacterium varium species can cause erosions in the colon similar to the lesions seen in patients with rectocolitis ${ }^{(37)}$.

Mutations in the nucleotide-binding oligomerization domain containing 2 (NOD2) gene that may be associated with the onset of Crohn's disease and variations of the interleukin receptor gene (IL-23) have been reported both in Crohn's disease and ulcerative rectocolitis ${ }^{(12,41)}$.

It has been shown that NOD2 dysfunction causes the translocation of enteric bacteria into the lamina propria, with alterations in cytokine expression. Mice with NOD-2 depletion have a significant increase in Bacteroides, Firmicutes and Bacillus in the terminal ileum and reduced capacity to eliminate more pathogenic bacterial species such as Helicobacter hepaticus ${ }^{(41)}$.

Studies comparing "germ-free" and colonized animals have shown that animals raised in sterile environments are less susceptible to experimental IBD and that the presence of commensal bacteria can trigger and/or exacerbate inflammatory bowel diseases ${ }^{(7,15)}$. It can be concluded that, in susceptible individuals, the inadequate immune response of the mucosa in relation to the gastrointestinal microbiota may result in Crohn's disease or ulcerative rectocolitis.

Similar to NOD2, TLRs (toll-like receptors) are also active participants in luminal interactions and studies have suggested that IBD may be associated with alterations in the selective expression of these receptors. Recent data have shown that levels of TLR-3 and 4 are altered in active IBD $^{(23)}$.

Defects in autophagy may also lead to some inability to maintain the stability of the intestinal microbiota. Studies have implicated the gene ATG1666L1, which codifies a protein involved in autophagosome formation, in the etiology of Crohn's disease ${ }^{(50)}$. Additionally, changes in intestinal permeability resulting in altered epithelial barrier function have been described ${ }^{(7)}$.

The concentration of bacteria in the colonic mucosa is substantially higher in patients with IBD than in healthy volunteers and this concentration also increases according to disease severity ${ }^{(42)}$. On the other hand, an experiment carried out on mice with suppressed IL-10 gene indicated that these animals developed ulcerative rectocolitis when they were colonized, but not when they were raised in a germ-free environment $t^{(15)}$.

It is known that the mucus has a protective function in the intestinal epithelium, forming an actual barrier and selectively preventing bacterial colonization. It has been shown that the mice with the thinnest and least sulphated intestinal mucus layer have a BOSI and are also more susceptible to developing rectocolitis ${ }^{(37)}$.

Such findings in studies in experimental animals and in patients with IBD suggest that the intestinal microbiota may play an important role in the pathogenesis of Crohn's disease and ulcerative rectocolitis $\mathrm{A}^{(50)}$. 


\section{Celiac disease}

In addition to the known immunological and genetic mechanisms, it is believed that environmental factors and the intestinal microbiota may have an effective participation in the pathophysiology of celiac disease ${ }^{(30,42)}$. Recent studies have shown the presence of intestinal dysbiosis in these patients (both in the untreated group and in those treated with the gluten-free diet) when compared to healthy individuals. In fact, some altered genes and/or their expression in celiac disease seem to play an active role in bacterial colonization and sensitization. On the other hand, dysbiosis can provoke the abnormal response to gluten (or other environmental factors that promote the disease) in genetically predisposed individuals ${ }^{(66)}$.

The gluten-free diet, alone, can influence the composition of the intestinal microbiota and thus constitutes a complicating factor in studies of celiac patients. Some researchers have shown that the gluten-free diet favors the reduction of beneficial bacteria, such as Bifidobacterium and Lactobacilli and the increase of gram negative bacteria, such as E. coli and Bacteroidetes ${ }^{(17,66)}$. To understand whether intestinal dysbiosis is the cause or the consequence of the disease, studies are required with healthy newborns from families at increased risk of the disease. These investigations might reveal which genes and genotypes are involved, as well as identify environmental factors capable of influencing the development of oral tolerance to gluten ${ }^{(17)}$. It is worth considering that it is possible that new probiotics and prebiotics will show to be of value in the treatment and even in the prevention of celiac disease.

\section{Gastrointestinal neoplasms}

There are a multiplicity of factors that influence the initiation of neoplasia and its subsequent course. Factors that may not be considered strictly carcinogenic may, nevertheless, affect the ultimate result in neoplasms. As such, several microbial species participate directly or indirectly in the genesis of many malignant neoplasms; according to conservative estimates, at least $15 \%$ of all cancer cases are attributable to infectious agents ${ }^{(10,42,53)}$. Very little is known about the contribution of the intestinal microbiota to the development of digestive neoplasms. Enteric microorganisms that can promote carcinogenesis through different mechanisms ${ }^{(1,42,68)}$ are: 1) Inflammation induction; 2) Increased cell proliferation; 3) Alteration in stem cell dynamics; 4) Production of some substances such as butyrate, capable of affecting DNA integrity and immune regulation.

Studies in experimental animals and in humans have identified effector species and/or interrelations between members of the microbial community of the stomach and colon, which increases the risk of developing malignant lesions in these organs. The several manipulation strategies of the microbiota or the host's immune response to these microorganisms may in the future prevent or even treat certain types of gastrointestinal neoplasms ${ }^{(1,68)}$.

\section{Esophageal cancer}

Although there is still little research on the effects of the microbiome on the development of esophageal cancer, it is possible to consider that alterations in the gastric microbiota may contribute to the increased incidence of esophageal adenocarcinoma - particularly those arising near the gastroesophageal junction ${ }^{(1)}$. Some studies have suggested that the esophageal endogenous microbiota differs between individuals with healthy esophageal mucosa and those with Barrett's esophagus ${ }^{(26,68)}$. It is worth mentioning that the studies in this area are still very incipient, but it is possible that the differentiation between the esophageal and gastric microbioma will constitute an important factor for future investigations.

\section{Gastric cancer}

Helicobacter pylori $(H$. pylori) is considered a type 1A carcinogen by the $\mathrm{WHO}^{(3)}$. It initiates the inflammatory cascade that progresses through chronic gastritis to intestinal metaplasia, gastric atrophy, and gastric cancer. Only a minority of infected patients, however, will develop cancer, and the bacterium is absent or minimally present in neoplastic lesions. Experimental studies in animals demonstrate that the interrelationships between $H$. pylori and non- $H$. pylori gastric microbiota represent a new challenge to the dogma of gastric carcinogenesis ${ }^{(3,68)}$.

In contrast to the current model of gastric carcinogenesis, which is focused on the interaction between different strains of $H$. pylori and the host's genetic factors, research has suggested a new hypothesis, where early interactions between this microorganism and the gastric mucosa can determine alterations in the gastric microbiota structure, which could result in gastric atrophy ${ }^{(1,56)}$. It is possible that the new microbiota that develops in the stomach in response to low acidity conditions effectively participates in gastric carcinogenesis $^{(3,56)}$.

\section{Colorectal cancer}

The culmination of multiple genetic and epigenetic events directly contributes to the pathogenesis of colon and rectal cancer $(\mathrm{CRC})^{(1)}$. The neoplasia is initiated by mutations in some tumor suppressor genes (APC, CTNNB1, p53) and oncogenes (KRAS) that transform healthy intestinal mucosa into adenoma and neoplasia ${ }^{(25)}$. Although the mutations in these genes already have defined roles in the development of $\mathrm{CRC}$, the events that lead to the acquisition of these mutations and epigenetic modifications are still unclear ${ }^{(68)}$. Recent studies have identified the intestinal microbiota and some environmental factors, such as diet and lifestyle, as potential promoters of CRC development ${ }^{(2)}$.

It is still unclear whether there are specific microorganisms that are particularly pathogenic and can directly participate in carcinogenesis or whether the process requires specific interactions between host tissues and the colonic microbiota and, probably, several mechanisms must be involved ${ }^{(1,25)}$. A future challenge will be to identify which microorganisms are effectively associated with adenocarcinoma development.

In vitro and in vivo studies indicate that high fiber intake can bring benefits to intestinal health and decrease the incidence of colorectal cancer. This is due to the fact that fibers are fermented by the colonic bacteria, later forming short-chain amino acids, and among them butyrate, which after being captured by the enterocytes, is used as a local source of energy ${ }^{(2)}$. Butyrate seems to be capable of inducing apoptosis and inhibiting the proliferation of neoplastic colon cells. The formation of a higher percentage of this substance is associated mainly with the action of anaerobic bacteria, such as Clostridium, Eubacterium and Fusobacterium ${ }^{(3,42,68)}$.

Analysis of the fecal microbiome of patients with CRC shows the increase of the Bacteroides species, decrease of butyrate-producing bacteria and increase of potentially pathogenic bacteria, such as the Fusobacterium species ${ }^{(25)}$. It has been demonstrated that the microbiota present in the neoplastic lesion differs from that found in the surrounding colonic mucosa (normal), with greater abundance of Coriobacteriaceae. It is probable that the alterations in the microbiota next to the neoplasms are associated to nutrient availability and other conditions created by the neoplastic cells themselves ${ }^{(1)}$. Future studies will investigate whether a state of dysbiosis precedes the development of adenomas and CRC. 
Experimental studies suggest that probiotics may inhibit CRC by interfering with the immune system and apoptosis, being able to modulate intestinal bacteria and their metabolism ${ }^{(2,25)}$. It has been observed that Lactobacillus johnsonii reduced the concentrations of Enterobacteria, modulating the immune response in patients with CRC. This effect was not described with strains of Bifidobacterium longum ${ }^{(6)}$. A clinical trial showed that strains of Lactobacillus casei were able to reduce tumor growth after 2 to 4 years of treatment ${ }^{(64)}$. Clinical trials in this area, however, are still quite limited by the small number of enrolled patients and a short follow-up period.

\section{CONCLUSION}

Alterations in the composition and function of the gastrointestinal microbiota (dysbiosis) have a direct impact on human health and seem to have an important role in the etiopathogenesis of several gastrointestinal diseases, whether inflammatory, metabolic, or neoplastic ones.

New studies on the interrelationship of the intestinal microbiota with the host will be essential to recognize the possible strategies of how to favorably handle the thousands of microorganisms that inhabit the digestive tract, promoting eubiosis and fighting the associated diseases.

\section{Authors' contributions}

Passos MCF designed the study, collected and analyzed data, drafted the manuscript, criticaly revised the article for important medical content and approved the final manuscript draft. MoraesFilho JP collected and analyzed data, critically revised the article for important medical content and approved the final manuscript draft.

Passos MCF, Moraes-Filho JP. Microbiota intestinal nas doenças digestivas. Arq Gastroenterol. 2017;54(3):255-62.

RESUMO - Contexto - Nos últimos anos, especialmente a partir do desenvolvimento de sofisticados estudos metagenômicos, as pesquisas acerca da microbiota intestinal se intensificaram, transformando de forma radical os nossos conhecimentos sobre o microbioma e sua relação com a manutenção da saúde e o desenvolvimento de doenças no ser humano. Evidências crescentes demonstram que uma alteração permanente da composição ou da função da microbiota (disbiose) pode alterar as respostas imunológicas, o metabolismo, a permeabilidade intestinal e a motilidade digestiva, promovendo, dessa maneira, um estado pró-inflamatório. Tais alterações podem comprometer, sobretudo, as funções imunes e metabólicas do hospedeiro, favorecendo o aparecimento de doenças como diabetes, obesidade, doenças digestivas, neurológicas, autoimunes e neoplásicas. Este artigo de revisão é uma compilação da literatura disponível sobre a formação do complexo ecossistema intestinal e seu impacto na incidência de doenças como obesidade, esteatohepatite não alcoólica, síndrome do intestino irritável, doença inflamatória intestinal, doença celíaca e neoplasias digestivas. Conclusão - Alterações na composição e função da microbiota gastrointestinal (disbiose) têm um impacto direto sobre a saúde humana e parecem ter um papel importante na patogênese de várias doenças gastrointestinais, sejam elas inflamatórias, metabólicas ou neoplásicas.

DESCRITORES - Microbiota. Microbioma gastrointestinal. Disbiose. Gastroenteropatias.

\section{REFERENCES}

1. Abreu MT, Peek RM Jr. Gastrointestinal Malignancy and the Microbiome. Gastroenterology. 2014;146:1534-46.

2. Akin H, Tözün NJ. Diet, microbiota, and colorectal cancer. Clin Gastroenterol. 2014;48:S67-9.

3. Amieva M, Peek RM Jr. Pathobiology of Helicobacter pylori-Induced Gastric Cancer. Gastroenterology. 2016;150:64-78.

4. Arrieta MC, Stiemsma LT, Amenyogbe N, Brown EM, Finlay B. The intestinal microbiome in early life: health and disease. Front Immunol. 2014;5:427.

5. Arumugam M, Raes J, Pelletier E, Le Paslier D, Yamada T, Mende DR, et al. Enterotypes of the human gut microbiome. Nature. 2011;473:174-80.

6. Azcárate-Peril MA, Sikes M, Bruno-Bárcena JM. The intestinal microbiota, gastrointestinal environment and colorectal cancer: a putative role for probiotics in prevention of colorectal cancer? Am J Physiol Gastrointest Liver Physiol. 2011;301:401-24

7. Becker $\mathrm{C}$, Neurath MF, Wirtz S. The Intestinal Microbiota in Inflammatory Bowel Disease. ILAR J. 2015;56:192-204.

8. Bennet SM, Ohman L, Simren M. Gut microbiota as potential orchestrators of irritable bowel syndrome. Gut Liver. 2015;9:318-31.

9. Biasucci G, Benenati B, Morelli L, Bessi E, Boehm G. Cesarean delivery may affect the early biodiversity of intestinal bacteria. J Nutr. 2008:138:1796S-1800S

10. Biedermann L, Rogler G. The intestinal microbiota: its role in health and disease. Eur J Pediatr. 2015;174:151-67.

11. Blaser MJ. The microbiome revolution. J Clin Invest. 2014;124:4162-5.

12. Boukercha A, Mesbah-Amroun H, Bouzidi A, Saoula H, Nakkemouche M, Roy M, et al. NOD2/CARD15 gene mutations in North Algerian patients with inflammatory bowel disease. World J Gastroenterol. 2015;21:7786-94.

13. Boursier J, Diehl AM. Nonalcoholic Fatty Liver Disease and the Gut Microbiome. Clin Liver Dis. 2016;20:263-75

14. Brahe LK, Astrup A, Larsen LH. Can We Prevent Obesity-Related Metabolic Diseases by Dietary Modulation of the Gut Microbiota? Adv Nutr. 2016;7:90-101.
15. Bringiotti R, Ierardi E, Lovero R, Losurdo G, Di Leo A, Principi M. Intestinal microbiota: The explosive mixture at the origin of inflammatory bowel disease? World J Gastrointest Pathophysiol. 2014;5:550-9.

16. Carroll IM, Ringel-Kulka T, Siddle JP, Ringel Y. Alterations in composition and diversity of the intestinal microbiota in patients with diarrhea-predominant irritable bowel syndrome. Neurogastroenterol Motil. 2012; 24:521-30.

17. Cenit MC, Olivares M, Codoñer-Franch P, Sanz Y. Intestinal Microbiota and Celiac Disease: Cause, Consequence or Co-Evolution? Nutrients. 2015;798:6900-23.

18. Chiodini RJ, Dowd SE, Davis B, Galandiuk S, Chamberlin WM, Kuenstner JT, et al. Crohn's disease may be differentiated into 2 distinct biotypes based on the detection of bacterial genomic sequences and virulence genes within submucosal tissues. Clin Gastroenterol. 2013;47:612-20.

19. Darfeuille-Michaud A, Boudeau J, Bulois P, Neut C, Glasser AL, Barnich N, et al. High prevalence of adherent-invasive Escherichia coli associated with ileal mucosa in Crohn's disease. Gastroenterology. 2004;127:412-21.

20. Dave M, Higgins PD, Middha S, Rioux KP. The human gut microbiome: current knowledge, challenges, and future directions. Transl Res. 2012;160:246-57.

21. David LA, Maurice CF, Carmody RN, Gootenberg DB, Button JE, Wolfe $\mathrm{BE}$, Diet rapidly and reproducibly alters the human gut microbiome. Nature. 2014:505:559-63.

22. Di Mauro A, Neu J, Riezzo G, Raimondi F, Martinelli D, Francavilla R, et al. Gastrointestinal function development and microbiota. Ital J Pediatr. 2013;39:15.

23. Fernandes P, MacSharry J, Darby T, Fanning A, Shanahan F, Houston A, et al. Differential expression of key regulators of Toll-like receptors in ulcerative colitis and Crohn's disease: a role for Tollip and peroxisome proliferator-activated receptor gamma? Clin Exp Immunol. 2016;183:358-68.

24. Ford AC, Quigley EM, Lacy BE, Lembo AJ, Saito YA, Schiller LR, et al. Efficacy of prebiotics, probiotics, and synbiotics in irritable bowel syndrome and chronic idiopathic constipation: systematic review and meta-analysis. Am J Gastroenterol. 2014;109:1547-61. 
25. Gagnière J, Raisch J, Veziant J, Barnich N, Bonnet R, Buc E, et al. Gut microbiota imbalance and colorectal cancer. World J Gastroenterol. 2016; 22:501-18.

26. Gall A, Fero J, McCoy C, Claywell BC, Sanchez CA, Blount PL, et al. Bacterial Composition of the Human upper Gastrointestinal tract Microbiome is dynamics and associated with Genomic Instability in a Barrett's Esophagus Cohort. PLoS One. 2015;10:e0129055.

27. Ghazalpour A, Cespedes I, Bennett BJ, Allayee H. Expanding role of gut microbiota in lipid metabolism. Curr Opin Lipidol. 2016;27:141-7.

28. Gibson PR, Sheperd SJ. Evidence-based dietary management of functional gastrointestinal symptoms: the FODMAP approach. J Gastroenterol Hepatol 2010;25:252-8.

29. Gkolfakis P, Dimitriadis G, Triantafyllou K. Gut microbiota and non-alcoholic fatty liver disease. Hepatobiliary Pancreat Dis Int. 2015;14:572-81.

30. Goulet $\mathrm{O}$. Potential role of the intestinal microbiota in programming health and disease. Nutr Rev. 2015;73:32-40.

31. Halvorson HA, Schlett CD, Riddle MS. Postinfectious irritable bowel syndrome--a meta-analysis. Am J Gastroenterol. 2006;101:1894-9.

32. Hartstra AV, Bouter KE, Bäckhed F, Nieuwdorp M. Insights into the role of the microbiome in obesity and type 2 diabetes. Diabetes Care. 2015;38:159-65.

33. Human Microbiome Project Consortium. Structure, function and diversity of the healthy human microbiome. Nature. 2012;486:207-14.

34. Iqbal S, Quigley EM. Progress in our understanding of the Gut Microbiome: Implications for the Clinician. Curr Gastroenterol. Rep. 2016;18:49-57.

35. Kaji I, Karaki S, Kuwahara A. Short-chain fatty acid receptor and its contribution to glucagon-like peptide-1 release. Digestion. 2014;89:31-6.

36. Kassinen A, Krogius-Kurikka L, Mäkivuokko H, Rinttilä T, Paulin L, et al. The fecal microbiota of irritable bowel syndrome patients differs significantly from that of healthy subjects. Gastroenterology. 2007:133:24-33.

37. Kato K, Ohkusa T, Terao S, Chiba T, Murakami K, Yanaka A, et al. Adjunct antibiotic combination therapy for steroid-refractory or -dependent ulcerative colitis: an open-label multicentre study. Aliment Pharmacol Ther. 2014;39:949-56.

38. Lacy BE, Chey WD, Lembo AJ. New and Emerging Treatment Options for Irritable Bowel Syndrome. Gastroenterol Hepatol. 2015;11:1-19.

39. Lacy BE, Mearin F, Chang L, Chey WD, Lembo AJ, Simrén M, et al. Bowel Disorders. Gastroenterology. 2016;150:1393-1407.

40. Lee KN, Lee OY. Intestinal microbiota in pathophysiology and management of irritable bowel syndrome. World J Gastroenterol. 2014;20:8886-97.

41. Long WY, Chen L, Zhang CL, Nong RM, Lin MJ, Zhan LL, et al. Association between NOD2/CARD15 gene polymorphisms and Crohn's disease in Chinese Zhuang patients. World J Gastroenterol. 2014; 20:4737-44.

42. Lynch SV, Pedersen O. The Human Intestinal Microbiome in Health and Disease N Engl J Med. 2016;375:2369-79.

43. Macfarlane GT, Macfarlane S. Bacteria, colonic fermentation, and gastrointestina health. J AOAC Int. 2012;95:50-60

44. Min YW, Rhee PL. The Role of Microbiota on the Gut Immunology. Clin Ther 2015;37:968-75

45. Mizock BA. Probiotics. Dis Mon 2015;61:259-290.

46. Mondot S, de Wouters T, Dore J, Lepage P. The human gut microbiome and its dysfunctions. Dig Dis. 2013;31:278-85.

47. Moraes-Filho JPP, Quigley EM. The intestinal microbiota and the role of probiotics in irritable bowel syndrome: a review. Arq Gastroenterol. 2015;52:331-7

48. Pimentel $\mathrm{M}$. The prevalence of small intestinal bacterial overgrowth in irritable bowel syndrome: IBS vs healthy controls (not historical definitions). Gut. 2008;57:1334-5
49. Pimentel M. Review article: potential mechanisms of action of rifaximin in the management of irritable bowel syndrome with diarrhoea. Aliment Pharmacol Ther. 2016;43:37-49.

50. Qiao YQ, Cai CW, Ran ZH. Therapeutic Modulation of the Gut Microbiota in IBD - More Questions to Be Answered. J Dig Dis. 2016;17:800-810.

51. Quigley EM. Microflora modulation of motility. J Neurogastroenterol Motil 2011;17:140-7.

52. Rajilić-Stojanović M, Biagi E, Heilig HG, Kajander K, Kekkonen RA, et al. Global and deep molecular analysis of microbiota signatures in fecal samples from patients with irritable bowel syndrome. Gastroenterology. 2011;141:1792-801.

53. Robles-Alonso V, Guarner F. Progress in the knowledge of the intestinal human microbiota. Nutr Hosp. 2013;28:553-7.

54. Saavedra JM, Dattilo AM. Early development of intestinal microbiota: implications for future health. Gastroenterol Clin North Am. 2012:41:717-31.

55. Schwille-Kiuntke J, Mazurak N, Enck P. Systematic review with meta-analysis: post-infectious irritable bowel syndrome after travellers' diarrhoea. Alimen Pharmacol Ther. 2015;41:1029-37.

56. Shimizu T, Marusawa H, Watanabe N, Chiba T. Molecular Pathogenesis of Helicobacter pylori-Related Gastric Cancer. Gastroenterol Clin North Am. 2015;44:625-38

57. Shukla R, Ghoshal U, Dhole TN, Ghoshal UC. Fecal Microbiota in Patients with Irritable Bowel Syndrome Compared with Healthy Controls Using Real-Time Polymerase Chain Reaction: An Evidence of Dysbiosis. Dig Dis Sci. 2015;60: 2953-62.

58. Simrén M, Barbara G, Flint HJ, Spiegel BM, Spiller RC, Vanner S, et al. Intestinal microbiota in functional bowel disorders: a Rome foundation report. Gut. 2013;62:159-76.

59. Sommer F, Backhed F. The gut microbiota-masters of host development and physiology. Nat Rev Microbiol. 2013;11:227-38.

60. Spiller R, Garsed K. Postinfectious irritable bowel syndrome. Gastroenterology. 2009;136:1979-88.

61. Strachan DP. Hay fever, hygiene, and household size. BMJ. 1989;299:1259-60.

62. Surana NK, Kasper DL. The yin yang of bacterial polysaccharides: lessons learned from B. fragilis PSA. Immunol Rev. 2012;245:13-26.

63. Thabane M, Kottachchi DT, Marshall JK. Systematic review and meta-analysis: The incidence and prognosis of post-infectious irritable bowel syndrome. Aliment Pharmacol. Ther. 2007;26:535-44.

64. Tsai TL, Li AC, Chen YC, Liao YS, Lin TH. Antimicrobial peptide m 2163 or m2386 identified from Lactobacillus casei ATCC 334 can trigger apoptosis in the human colorectal cancer cell line SW480. Tumour Biol. 2015;36:3775-89.

65. Turnbaugh PJ, Ley RE, Mahowald MA, Magrini V, Mardis ER, Gordon JI. An obesity associated gut microbiome with increased capacity for energy harvest. Nature. 2006;444:1027-31

66. Wacklin P, Laurikka P, Lindfors K, Collin P, Salmi T, Lähdeaho ML, et al Altered duodenal microbiota composition in celiac disease patients suffering from persistent symptoms on a long-term gluten-free diet. Am J Gastroenterol. 2014;109:1933-41

67. Wallace TC, Guarner F, Madsen K, Cabana MD, Gibson G, Hentges E, et al Human gut microbiota and its relationship to health and disease. Nutr Rev. 2011;69:392-403.

68. Zitvogel L, Galluzzi L, Viaud S, Vétizou M, Daillère R, Merad M, et al. Cance and the gut microbiota: an unexpected link. Sci Transl Med. 2015;7:271ps1. 